Purdue University

Purdue e-Pubs

Other Nanotechnology Publications

Birck Nanotechnology Center

$8-18-2008$

\title{
OMEN an atomistic and full-band quantum transport simulator for post-CMOS nanodevices
}

Mathieu Luisier

Purdue University - Main Campus

Gerhard Klimeck

Purdue University - Main Campus, gekco@purdue.edu

Follow this and additional works at: http://docs.lib.purdue.edu/nanodocs

Luisier, Mathieu and Klimeck, Gerhard, "OMEN an atomistic and full-band quantum transport simulator for post-CMOS nanodevices" (2008). Other Nanotechnology Publications. Paper 142.

http://docs.lib.purdue.edu/nanodocs/142

This document has been made available through Purdue e-Pubs, a service of the Purdue University Libraries. Please contact epubs@purdue.edu for additional information. 


\title{
OMEN an atomistic and full-band quantum transport simulator for post-CMOS nanodevices
}

\author{
M. Luisier and G. Klimeck \\ Network for Computational Nanotechnology, Purdue University, West Lafayette, IN 47907, USA \\ E-mail: mluisier@purdue.edu
}

\begin{abstract}
The technology computer aided design of nanometer-scaled semiconductor devices requires appropriate quantum-mechanical models that capture the atomic granularity of the simulation domain. The recently developed nanodevice simulator OMEN fulfills this condition. It is able to treat two- and three-dimensional transistor structures in a full-band framework using the semi-empirical $s p^{3} d^{5} s^{*}$ tight-binding model. In this formalism each atom of the device is represented by a set of ten orbitals leading to multi-band and open-boundary Schrödinger equations that have to be solved thousands of times. To improve its computational efficiency OMEN has four levels of parallelism that make it run on the largest available supercomputers.
\end{abstract}

\section{INTRODUCTION}

Within the next five to ten years the semiconductor industry will face the greatest challenge in its history. The conventional planar bulk MOSFETs will reach their physical and technical limit and the continuation of Moore's law will depend on novel and original device structures. For example the feasibility of silicon-on-insulator ultra-thin-body (UTB) and multi-gate nanowire (NW) field-effect transistors (FETS) has already been demonstrated by several groups [1], [2], [3].

Modeling such devices requires approaches beyond the classical drift-diffusion and effective mass approximations. The treatment of strong quantization effects as well as the atomic granularity and dimensions of ultra-scaled transistors is demanding state-of-the art physical models. For that purpose we have developed OMEN [4], [6], [7] the first atomistic and full-band quantum transport simulator designed for postCMOS transistors like UTB and NW FETs that can treat realistically extended and gated devices. A brief summary of its simulation capabilities is presented in this paper.

\section{MethoD}

OMEN is a two- and three-dimensional SchrödingerPoisson solver based on the $s p^{3} d^{5} s^{*}$ semi-empirical tightbinding method [8]. This bandstructure model has been chosen for (i) its ability to reproduce the principal bulk characteristics of electrons and holes, (ii) its straight-forward extension to nanostructures, and (iii) its atomic description of the simulation domain. Carrier and current densities are obtained by injecting electrons and holes at different momentum and energies into the device and by solving the resulting system of equations in the Wave Function (WF) or in the Nonequilibrium Green's Function (NEGF) formalism [4]. The treatment of the open-boundary conditions (OBCs) deserves a special attention since their calculation can generate most of the computational burden if standard techniques such as iterative solvers or generalized eigenvalue problems are used. In OMEN the OBCs are obtained from a shift-and-invert procedure resulting in a normal eigenvalue problem which is at least one order of magnitude faster than the usual approaches [5].

The WF and the NEGF formalisms give the density-ofstates (DOS) and the transmission probability (TE) from one contact of the device to the other(s) as function of the injected momentum and energy. The carrier and current densities are calculated by integrating the DOS and TE, respectively and they are self-consistently coupled to the solution of a $2 \mathrm{D}$ or 3D Poisson equation. For that purpose a finite-element grid is constructed and the electron and hole charges coming from the tight-binding calculation are represented by delta-functions placed on node positions.

The core operation of OMEN is the calculation of the openboundary conditions and of the carrier and current densities using either NEGF or WF. This is repeated for each energy, momentum, self-consistent Poisson iteration, and bias point. In a typical 2D UTB simulation $N_{E}=500$ to 2000 energy points and $N_{k}=10$ to 20 momentum points have to be included for each Poisson iteration and bias point to get accurate results. In other words the Schrödinger equation has to be solved at least 5000 times per bias point. Even if this can be achieved in a couple of seconds the calculation of one single bias point without self-consistency will take hours on a single processor. However, more and more researchers have access to clusters composed of hundreds of processors. To benefit from this resources the computation of the bias points, the energy and momentum integrations, and the spatial decomposition of the simulation domain have been parallelized so that a single simulation can run on a number of processors $N_{C P U}$ comprised between $O\left(10^{3}\right)$ and $O\left(10^{4}\right)$ with an almost perfect speed-up factor of $N_{C P U}$.

\section{RESULTS}

Typical device structures that OMEN can handle are shown in Fig. 1 and comprise nanowire field-effect transistors with any cross section shape (square, circular, triangular, hexagonal, $\cdots)$, gate architecture (all-around, single, double, or triple), and transport direction $(<100\rangle,<110\rangle,<111\rangle,<112>$, ...) as well as ultra-thin-body FETs with single- or doublegate and any configuration of surface orientation and transport 

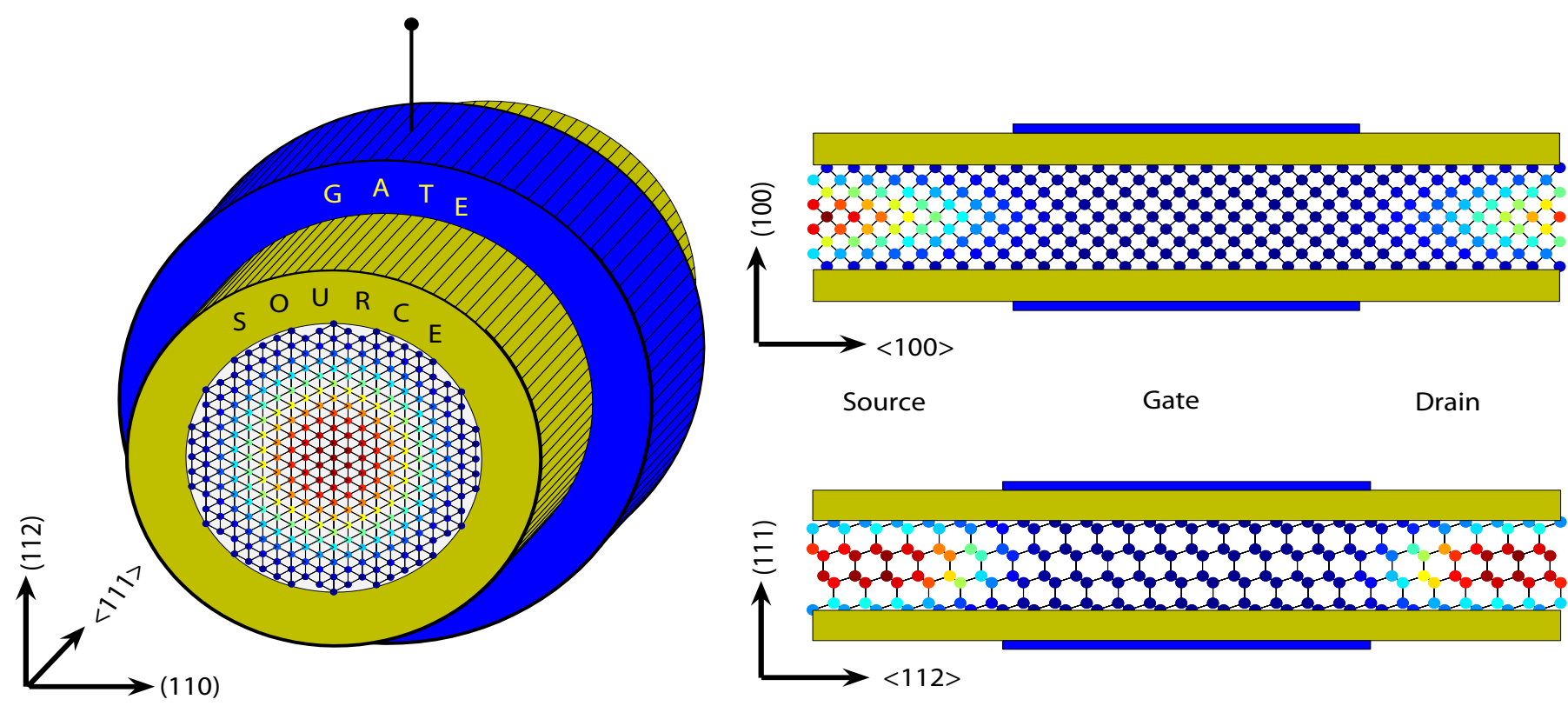

Source

Gate

Drain

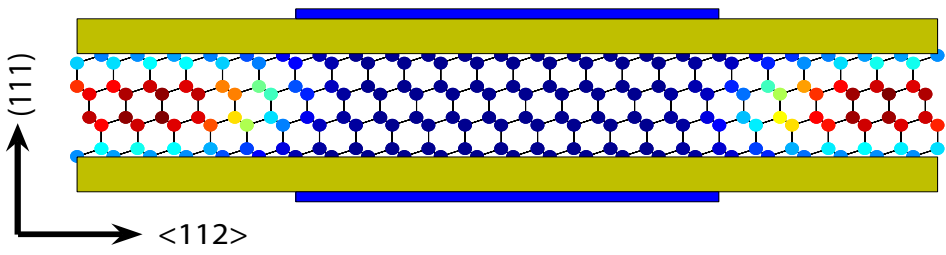

Fig. 1. Schematic view of a circular gate-all-around nanowire (left) and of two double-gate (right) field-effect transistors (FETs) with different transport directions $<>$ and surface orientations (). The nanowire and the ultra-thin-body are surrounded by oxide layers of thickness $t_{o x}$ that isolate them from the metallic gate contacts. The dots represent atoms that are colored according to the carrier distribution. Red atoms depict a high carrier density, blue atoms a low density.

direction. All the channel atoms are represented in the tightbinding formalism. The oxide layers surrounding the channel do not participate to the transport calculation due to a poor parametrization of the material and an insufficient knowledge of their coupling to the semiconductor. The oxide layers are modeled in the Poisson equation as perfect insulators characterized by their relative permittivity $\epsilon_{r}$ and an infinite band gap. Hence, the electrons are confined in the channel and do not penetrate into the oxide as illustrated in Fig. 1.

Different semiconductor materials have been parametrized, among them $\mathrm{Si}, \mathrm{Ge}, \mathrm{GaAs}$, InAs, AlAs, or SiGe. Ternary alloy semiconductors like InGaAs and AlGaAs are treated either as virtual crystals or as atomistically and randomly disordered entities [9]. The parameters are obtained by fitting the band gaps and effective masses of the bulk bandstructure at points of high symmetry. These semi-empirical parameters are assumed unchanged for nanostructures where the surface atoms are passivated.

Current characteristics and internal quantities such as the transmission coefficient, the density-of-states, the carrier density, and the electrostatic potential can be investigated for nand p-doped transistors. For example in Fig. 2 the logarithmic and ballistic transfer characteristics $I_{d}-V_{g s}$ of n-doped gate-all-around nanowire transistors with a diameter $d=4 \mathrm{~nm}$ and a gate length $L_{g}=10 \mathrm{~nm}$ are compared for transport along different crystal axis. Similarly in Fig. 3 the ballistic transfer characteristics of n-doped and p-doped double-gate UTB FETS are shown. The six devices have the same dimensions and surface orientation (100), but different transport directions $(<100\rangle,<110\rangle$, and $<111\rangle)$. Such comparisons are straight-forward in a full-band model, but require lot of approximations in effective-mass-like approaches.

OMEN offers a good insight into the internal quantities of the simulated devices as illustrated in Fig. 4 and 5. The maximum value of the drain current in Fig. 2 depends on the transport direction. To understand this behavior the corresponding transmission coefficients from the source to the drain contact in Fig. 4 can be investigated. It is observed that the first current channels open for the nanowire with transport along $<110>$. It is followed by the $<100>$ nanowire that counts two times more channels. The two effects (energy turn-on and number of channels) more or less compensate each other so that the $<110\rangle$ and $<100>$ currents are about the same.

Another important transistor characteristics is its subthreshold swing or the amount of gate voltage change necessary to increase (or decrease) the drain current by one order of magnitude. This information is directly related to the quality of the control one has over the potential barrier induced by the gate contact. Fig. 5 shows the variation of the potential barrier height for two gate voltages and the resulting inversion charges in the channel for a p-doped UTB FET. Ideally a variation of the gate voltage of $\delta V_{g}$ would change the barrier height by $q \cdot \delta V_{g}$ where $q$ is the elementary charge.

The transport in OMEN is not restricted to its ballistic component, but elastic scattering like surface roughness [6] and alloy disorder [9] can be fully taken into account due to the atomic nature of the simulation domain. For a given device configuration one can study the most advantageous transport direction and surface orientation with respect to ON- and OFFcurrent, subthreshold swing, drain-induced barrier lowering, source-to-drain tunneling, transient time, and sensitivity to surface roughness. 


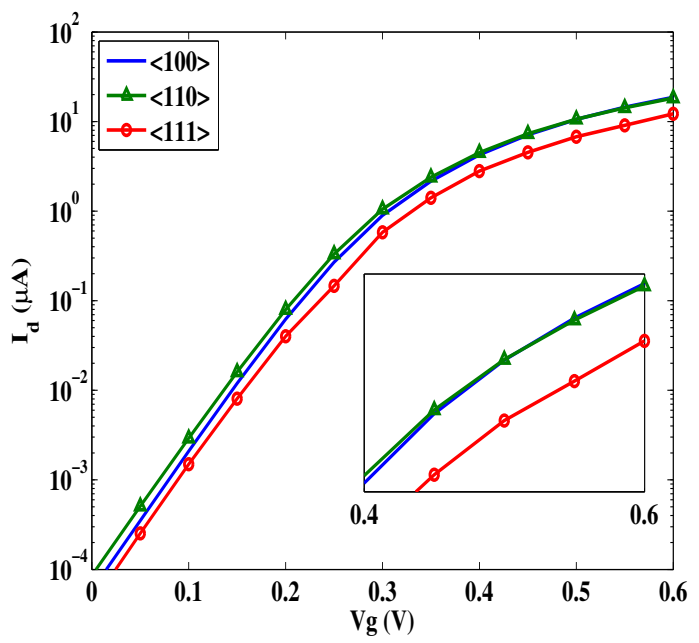

Fig. 2. Transfer characteristics $I_{d}-V_{g s}\left(V_{d s}=0.6 \mathrm{~V}\right)$ of n-doped Si gate-all-around nanowire FETs (diameter $d=4 \mathrm{~nm}$, gate length $L_{g}=10 \mathrm{~nm}$ ) with transport along the $\left.<100\right\rangle$, the $<110>$, and the $<111>$ crystal axis.

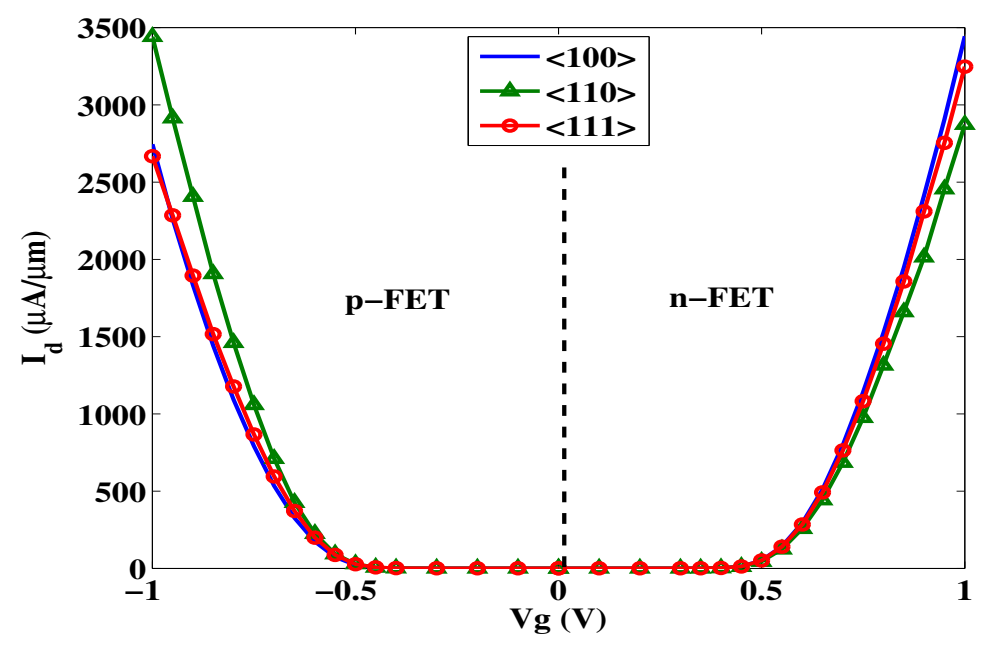

Fig. 3. Transfer characteristics $I_{d}-V_{g s}\left(V_{d s}= \pm 1.0 \mathrm{~V}\right)$ of n- (right) and p- (left) doped Si double-gate ultra-thin body (DG UTB) FETS (body thickness $t_{S i}=4.9 \mathrm{~nm}$, gate length $L_{g}=22$ $\mathrm{nm})$ with (110) as surface orientation and transport along the $\langle 100\rangle$, the $\langle 110\rangle$, and the $<111>$ crystal axis.

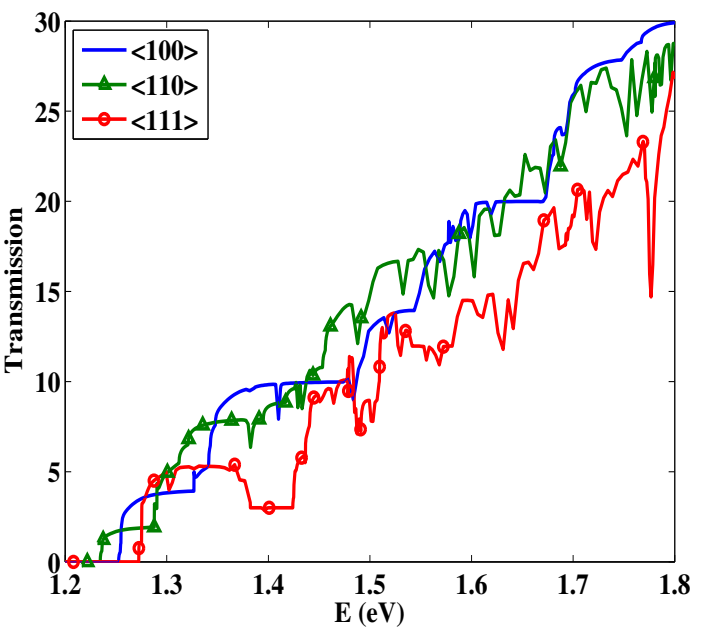

Fig. 4. Transmission coefficient through the same gate-all-around nanowire FETs as in Fig. 2 at $V_{g s}=V_{d s}=0.6 \mathrm{~V}$. The energy reference $E=0.0 \mathrm{eV}$ is the top of the bulk valence band.

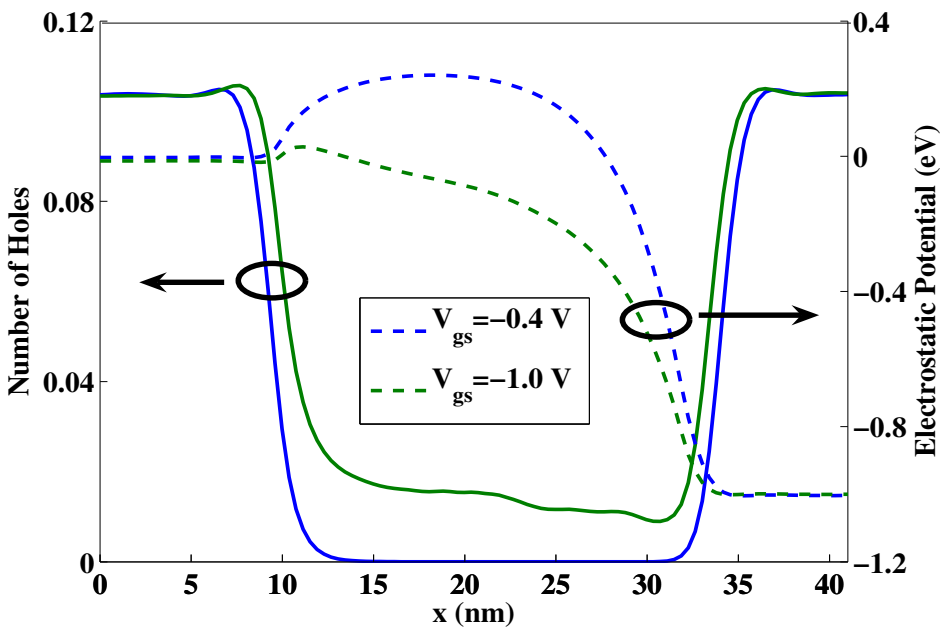

Fig. 5. Number of holes (solid lines) and inverse average electrostatic potential (dashed lines) along the transport direction of the $<110>/(110)$ DG UTB simulated in Fig. 3. Results were obtained at $V_{d s}=-1.0 \mathrm{~V}$.
The multi-level parallel implementation of OMEN and the optimization of its numerical algorithm make the simulation of NW with a cross section up to $22 \mathrm{~nm}^{2}$ and UTB with a body thickness up to $10 \mathrm{~nm}$ and a gate length of $L_{g}=40$ $\mathrm{nm}$ possible. For a realistic UTB structure designed according to the $22 \mathrm{~nm}$ technology node specifications an almost ideal scaling of the simulation walltime up to 32768 processors can be demonstrated [10]. In Fig. 6 the walltime measured for the simulation of a circular nanowire FET (green curve with triangle, diameter $d=3 \mathrm{~nm}$, gate length $L_{g}=15 \mathrm{~nm}$ ) and a double-gate UTB FET (blue curve with crosses, body thickness $t_{\text {body }}=4.9 \mathrm{~nm}$, gate length $L_{g}=22 \mathrm{~nm}$ ) are reported as function of the number of processors used. The dashed line indicates the ideal scaling slope.

\section{CONCLUSION}

At the moment no other full-band and atomistic quantum transport simulator offers such physical capabilities, computational efficiency, and support in developing nanotransistors as OMEN. Gate leakage currents, surface reconfiguration, discrete doping atoms, and dissipative electron-phonon scattering are planned for future extensions.

\section{ACKNOWLEDGMENT}

This work was partially supported by NSF grant EEC0228390 that funds the Network for Computational Nanotechnology, by NSF PetaApps grant number 0749140 , and by NSF through TeraGrid resources provided by TACC. nanohub.org computer resources were also used. 


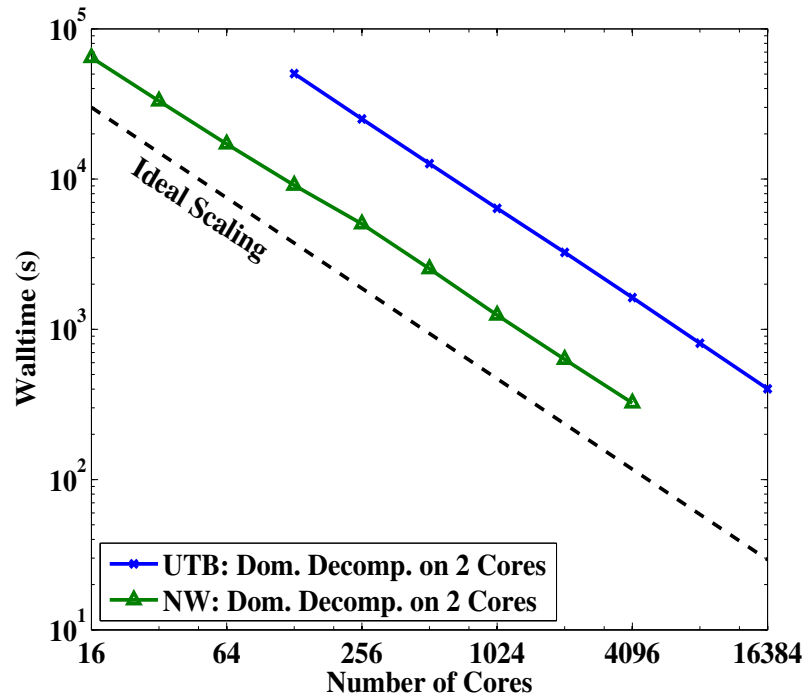

Fig. 6. OMEN scaling performance for the computation of the transfer characteristics $I_{d}-V_{g s}$ of a UTB (same structure as in Fig. 3, blue curve with crosses) and a circular nanowire (diameter of $3 \mathrm{~nm}$, green curve with triangles) field-effect transistor. The four levels of parallelism of OMEN are turned on for the UTB case ( 8 bias points, 16 momentum points, about 1400 energy points, and domain decomposition on 2 cores). The nanowire simulation requires three levels of parallelism (16 bias points, no momentum, about 540 energy points, and domain decomposition on 2 processors).

\section{REFERENCES}

[1] Y. Cui, L. J. Lauhon, M. S. Gudiksen, J. Wang, and C. M. Lieber, "Diameter-controlled synthesis of single-crystal silicon nanowires", Appl. Phys. Lett. vol. 78, 2214, 2001.

[2] S. D. Suk et al., "Investigation of nanowire size dependency on TSNWFET", IEDM Tech. Dig. 2007, pp. 891-894, 2007.

[3] B. Doris et al., "Extreme scaling with ultra-thin Si channel MOSFETs", IEDM Tech. Dig. 2002, pp. 267-270, 2002.

[4] M. Luisier, G. Klimeck, A. Schenk, and W. Fichtner, "Atomistic Simulation of Nanowires in the $s p^{3} d^{5} s^{*}$ Tight-Binding Formalism: from Boundary Conditions to Strain Calculations", Phys. Rev. B, vol. 74, 205323, 2006.

[5] M. Luisier and A. Schenk, "Atomistic Simulation of Nanowire Transistors", J. of Comp. and Theoretical Nanoscience, vol. 5, pp. 1031-1045, 2008.

[6] M. Luisier, A. Schenk, and W. Fichtner, "Three-Dimensional Full-Band Simulations of Si Nanowire Transistors", IEDM Tech. Dig. 2006, pp. 811814, 2006.

[7] M. Luisier, A. Schenk, and W. Fichtner, "Full-band atomistic study of source-to-drain tunneling in $\mathrm{Si}$ nanowire transistors", Proc. of Int. Conf. on Simulation of Semiconductor Processes and Devices (SIS$P A D)$, pp. 221-224, 2007.

[8] T. B. Boykin, G. Klimeck, and F. Oyafuso, "Valence band effective-mass expressions in the $\mathrm{sp}^{3} \mathrm{~d}^{5} \mathrm{~s}^{*}$ empirical tight-binding model applied to a $\mathrm{S}$ and Ge parametrization", Phys. Rev. B, vol. 69, 115201, 2004.

[9] T. B. Boykin, M. Luisier, A. Schenk, N. Kharche, and G. Klimeck, "The electronic structure and transmission characteristics of disordered $\mathrm{AlGaAs}$ nanowires, IEEE Trans. on Nanotech. vol. 6, pp. 43-47 2007.

[10] M. Luisier and G. Klimeck, "A multi-level parallel approach to the quantum mechanical simulation of nanoelectronic devices", unpublished. 